\title{
Assessment of melanoma risk in acquired melanocytic nevi using digital dermoscopic and 3- point checklist score
}

\author{
Göknur Özaydın Yavuz ${ }^{1 *}$, Necmettin Akdeniz ${ }^{2}$, İbrahim Halil Yavuz ${ }^{1}$, Ömer Çalka ${ }^{3}$, Serap Güneş \\ Bilgili $^{1}$ \\ ${ }^{1}$ Department of Dermatology, Van Yuzuncu Yil University Faculty of Medicine, Van, Turkey \\ 2Department of Dermatology, İstanbul Medeniyet University Faculty of Medicine, Göztepe Research and Training \\ Hospital, İstanbul, Turkey \\ ${ }^{3}$ Free Dermatologist, Ankara, Turkey
}

\begin{abstract}
Among skin cancers if not diagnosed early the highest death rate is for malign melanoma. Many studies has showed that multipl nevi lead to an increased risk of melanoma. It is a good option to take photos of patients in periodic intervals. In our study, we calculated the risk of melanoma in patients with acquired melanocytic nevi with 3-point checklist and digital dermoscopy and we aimed to compare the results and their advantages to each other.

We enrolled 300 patients with acquired melanocytic nevi in our study. Cases we enrolled in our study were recorded in the pre-prepared patient follow-up form. In this study Fotofinder HD (Germany) digital dermoscopy instruments were used. Lesions that were regarded as malign by 3-point scoring method were excised and histopathologically evaluated. In statistical calculations significance level of $5 \%$ was used and calculations were made with SPSS (ver: 13) statistical package program.

157 of our patients were male (52.3\%) and $143(47.7 \%)$ were female. Sensitivity of digital dermoscopy was determined as $86 \%$ according to the 3 -point checklist. 6 Of the 300 patients were diagnosed as malign melanoma. Sensitivity of the 3point checklist was determined as $97 \%$.

In our study, the results of 3-point checklist were detected higher than previous studies. Since digital dermoscopy is easy to use, this method is convenient especially for inexperienced physicians in the diagnosis of malignancy. But dermoscopic algorithms remain as the first choice for specialist physicians.
\end{abstract}

Key Words: Acquired melanocytic nevi, malign melanoma, digital dermoscopy

\section{Introduction}

Melanocytic nevi are benign melanocyte proliferations. They are well demarcated macula or papilla that may have the same color with the skin or may have reddish-brown, brown, or black color. Melanocytic nevi may be congenital or acquired. In white race, almost every individual have approximately 20 nevi on average (1).

In a typical life cycle melanocytic nevi develop after infancy, reach a peak number in the second and third decades of life, and disappear in the seventh to ninth decades of life. In individuals with advanced age, these lesions are rare and should raise a suspicion (2,3). Even if completely ordinary, an increased number of nevi is associated with an increased risk of melanoma. Of all melanomas, nearly $25 \%$ develop on a background of melanocytic nevi. However, it is impossible to foretell which nevi will pose an increased risk and removal of all melanocytic nevi is unfeasible (1-4). Dermoscopy and histological examination form the basis of the diagnosis and follow up of melanocytic nevi. A number of different computer software have been developed to be used in combination with digital dermoscopy to acquire, interpret, and store clinical and dermoscopic images. In a patient with multiple atypical nevi, it may not be possible to completely rule out a suspicious melanoma based on clinical or dermoscopic examination, despite a low level of clinical suspicion. In such cases, rather than relying on the physician's memory or clinical photographs, which are frequently difficult to use or retrieve, it may be more plausible to use serial dermoscopic images, since they allow comparison, calculation of dermoscopic scores, and effectively document the alterations $(3,5,6)$. In this study, 
digital dermoscopy as well as the 3-point check list scores have been evaluated in a total of 300 individuals attending to our dermatology outpatient unit with suspicious melanocytic nevi.

\section{Materials and Methods}

A total of 300 patients with suspicious melanocytic lesions attending to the Dermatology Outpatient Unit, Medical Faculty of Van Yuzuncu Yil University have been enrolled. Name/family name of the patient, gender, occupation, address and contact information, skin type, localization of the lesions, and diameter of the lesions were recorded in patient follow-up forms specifically designed for the study. The study protocol was approved by the Ethics Committee for Clinical and Laboratory Investigations.

For study purposes, a HD (Germany) digital dermoscopy device was used (Figure 1). Images were acquired with a Medicam 500 video camera with a dermoscopy lens at the tip that provided a 20 to $50 \mathrm{x}$ magnification power. The camera has a ccd-chip equipped with $1 / 4470.000$ pixels; optic zoom 18x, and lens size of 4.1-73.8 mm. During the digital image acquiring procedures, the skin area of interest was wiped with alcohol in order to smoothen the skin surface, provide deeper light penetration through reduced reflections, and therefore to improve the image quality. The fundamental characteristics of the digital dermoscopy device are based on ABCD scoring system. A digitally identified score exceeding 0.50 is considered to signify malignancy.

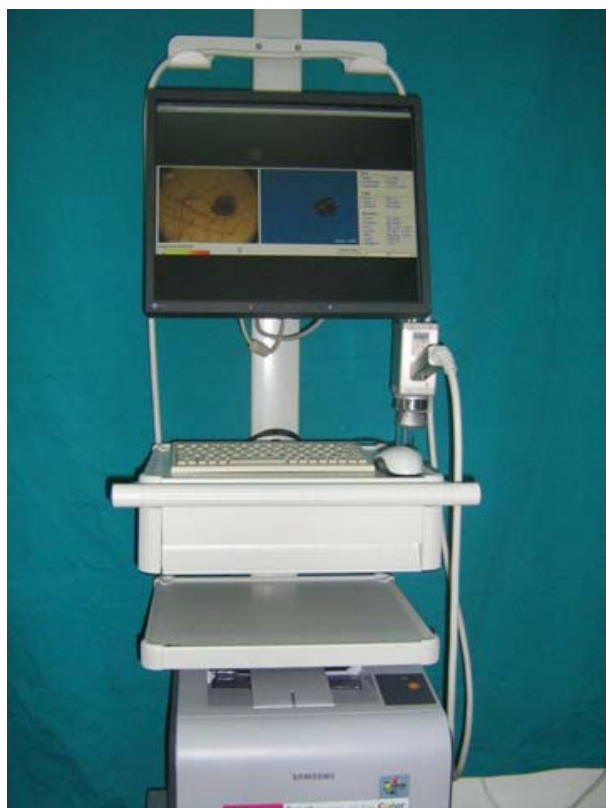

Fig. 1. Appearance of the used digital dermoscopy device.
In digitally photographed images, melanocytic nevi were also assessed according to the 3-point check list. Lesions with a score of 2 or higher were considered malign. Thus, digital dermoscopy scores and 3-point checklist scores were evaluated in combination. Lesions considered malign according to the 3-point scoring system were excised for histopathological examination.

The association between categorical variables was tested using the chi-square test. Also, for comparison of diagnostic test measures between 3 point scoring system and the digital test results (sensitivity, specificity, positive predictive value, negative predictive value, etc.), the level of significance was set at 5\%, and all data analyses were performed using SPSS (version 13) statistical software package. For independent groups, t test was used.

\section{Results}

There were 157 male (52.3\%) and 143 female $(47.7 \%)$ participants. The age range in male and female subjects was 10 to 80 and 12 to 69, respectively. The mean age in male and female participants were $31.78 \pm 1.109$ and $31.27 \pm 1.005$ years, with an overall mean age of $31.54 \pm 0.75$ years. Age and gender were evenly distributed across the study population (Table 1). The differences of nevi was not significantly different between the two sexes $(p>0.05)$ The skin type in our patients was 2-3-4, with the latter type being the most frequent $(77.3 \%)$. There were no significant gender differences with regard to skin type $(p>0.05)$. When the body distribution of malignant and benign lesions were examined using digital dermoscopy, $50 \%$ of the lesions localized in the leg and hip regions were malignant, while $87.5 \%$ of the abdominal lesions were considered benign. Irrespective of lesion localization, $18 \%$ of all lesions were considered malignant based on digital dermoscopy.

Table 1. Differences of patients according to age and gender.

\begin{tabular}{lcccccc}
\hline \multicolumn{7}{c}{ Age } \\
\hline Gender & $\mathrm{n}$ & Mean & SD & Min. & Max. & $\mathrm{p}$ \\
\hline Male & 157 & 31.78 & 13.89 & 10 & 80 & \\
Female & 143 & 31.27 & 12.02 & 12 & 69 & .731 \\
\hline Total & 300 & 31.54 & 13.02 & 10 & 80 &
\end{tabular}

SD: Standard Deviation 
An examination of the nevi with the 3-point checklist according to lesion localization suggested that many nevi in the legs and abdominal lesion were benign. The localization with the highest frequency of malign lesions was the chest region $(16.7 \%)$.

The skin type most frequently associated with malignant lesions based on 3-point checklist scores was type 2 (Table 2). Comparison of digital dermoscopy and 3-point checklist showed a sensitivity and a specificity of $93 \%$. Table 3 shows the result of assessments based on digital dermoscopy and 3-point checklist. The 3-point checklist was found to have high specificity as assessed by the results of histopathological examination (Table 4).

The sensitivity of the 3-point checklist according to histopathology result was $97 \%$. The false positivity rate was $3 \%$, i.e. patients with a negative (benign) histopathology result despite having a positive (malignant) 3-point checklist assessment.
The false negativity rate was $3 \%$, i.e. patients with a positive (malignant) histopathology result, despite a negative (benign) 3-point checklist assessment.

\section{Discussion}

Melanocytic nevi are benign tumors arising from nevus cells that derive from melanocytes. Of all cutaneous melanoma cases, 15 to $85 \%$ are known to originate from melanocytic nevi $(5,6)$.

In a study by Dennis et al. (7) melanocytic nevi were more frequently found among men, which was related to the duration of exposure to the ultraviolet light. In another study, men had a higher incidence of melanocytic nevi until age 39, followed by equal gender distribution after that age (8). Also, 32 patients included in a study, 53\% and $47 \%$ were male and female, respectively (8). In our study, there were 157 male (52.3\%) and 143 female patients (47.7\%), with no significant

Table 2. Three-point checklist scores according to skin type

\begin{tabular}{llccc}
\hline & & \multicolumn{2}{c}{3 -point checklist } & \multirow{2}{*}{ Total } \\
\cline { 3 - 4 } & & Benign & Malignant & \\
\hline Skin type & No. & 7 & 1 & 8 \\
& \% Within the skin type & $87.5 \%$ & $12.5 \%$ & $100.0 \%$ \\
& $\% 3$-point checklist & $2.5 \%$ & $6.7 \%$ & $2.7 \%$ \\
& $\%$ Total & $2.3 \%$ & $.3 \%$ & $2.7 \%$ \\
& No. & 59 & 1 & 60 \\
& $\%$ Within the skin type & $98.3 \%$ & $1.7 \%$ & $100.0 \%$ \\
& $\%$ 3-point checklist & $20.7 \%$ & $6.7 \%$ & $20.0 \%$ \\
& $\%$ Total & $19.7 \%$ & $.3 \%$ & $20.0 \%$ \\
& No. & 219 & 13 & 232 \\
& $\%$ Within the skin type & $94.4 \%$ & $5.6 \%$ & $100.0 \%$ \\
& $\%$ 3-point checklist & $76.8 \%$ & $86.7 \%$ & $77.3 \%$ \\
& $\%$ Total & $73.0 \%$ & $4.3 \%$ & $77.3 \%$ \\
& No. & 285 & 15 & 300 \\
& $\%$ Within the skin type & $95.0 \%$ & $5.0 \%$ & $100.0 \%$ \\
\% 3-point checklist & $100.0 \%$ & $100.0 \%$ & $100.0 \%$ \\
& \% Total & $95.0 \%$ & $5.0 \%$ & $100.0 \%$ \\
\hline
\end{tabular}

Chi-square $\left(\boldsymbol{\chi}^{2}\right)=0,28$

Table 3. Evaluation of digital dermatoscopy according to 3-point checklist

\begin{tabular}{|c|c|c|c|c|}
\hline & & \multicolumn{2}{|c|}{ 3-point checklist } & \multirow{2}{*}{ Total } \\
\hline & & Benign & Malignant & \\
\hline Digital & Benign & 245 & 1 & 246 \\
\hline Dermoscopy & Malign & 40 & 14 & 54 \\
\hline Total & & 285 & 15 & 300 \\
\hline
\end{tabular}

Table 4. Evaluation of pathology results according to 3-point checklist.

\begin{tabular}{lcccc}
\hline & & \multicolumn{2}{c}{ 3-point checklist } & \multirow{2}{*}{ Total } \\
\cline { 3 - 4 } & & Benign & Malignant & \\
\hline \multirow{2}{*}{ Pathology } & Benign & 285 & 9 & 294 \\
& Malign & 0 & 6 & 6 \\
\hline \multirow{2}{*}{ Total } & & 285 & 15 & 300 \\
\hline
\end{tabular}


difference between the two genders. In our patient population a high incidence of skin cancer is not surprising when one considers their residency in a geographical localization of high altitude, sunlight exposure, as well as higher prevalence of xeroderma pigmentosum. Dermoscopy is a noninvasive technique that allows a better visualization of the surface and undersurface characteristics of pigmented skin lesions with the aid of a light source and magnifier. With the naked eye, only $65 \%$ of the cutaneous melanomas can be diagnosed, while the rate of accurate diagnosis may reach up to $95 \%$ with dermoscopy (6). Therefore, although the rate of diagnostic accuracy has not reached $100 \%$, currently dermoscopy represents one of the most important means to differentiate melanomas from benign melanocytic nevi (8).

In 1994, Stolz et al. (9) introduced the ABCD system for identification of malignant lesions using dermoscopy. In this algorithm several characteristics of the lesion such as asymmetry, border characteristics, color, and differential structural components are assessed. A total score termed as total dermoscopy score is obtained after individual assessment of the lesions. As such, a total score exceeding 5.45 is considered to show the presence of malignancy. In the study by Stolz et al. (9) the diagnostic sensitivity and specificity reported for this approach are $92 \%$ and 97.95 , respectively. Since the method involves the use of only four criteria for assessments, a training could be easily provided and the technique could be readily applied in the current study. It represents a particularly suitable technique for physicians without adequate expertise (10). The fotofinder dermoscopy device used for patient assessment in this study principally operates according to the $A B C D$ criteria with automatic scoring. Of the 300 atypical melanocytic nevi examined, 54 were considered malign. Most of these lesions were elevated from the skin. Previous reports suggested higher rates of malignancy detection, particularly in elevated lesions or in lesions with an increased diameter, while flat macular or patch-like lesions tended to yield better results. Therefore, we hold the view that this device is more likely to fail diagnostically for elevated lesions. In our study, since the number of cases categorized as having malignant lesions with this device was high, no histopathological correlation could be performed. However, histopathological assessments were performed for lesions that have been categorized as malignant with the 3-point checklist score. Based on these evaluations, digital dermoscopy was found to have a sensitivity of $86 \%$, which is slightly higher as compared to previous reports.

Carli et al. (11) examined a total of 168 melanocytic lesions clinically, dermoscopically, and histopathologically. For dermoscopy, a pattern analysis was carried out. Malignancy detection rates with dermoscopy and clinical observation were $45 \%$ and $28 \%$, respectively, concluding that pattern analysis was an effective diagnostic approach. In a similar study by Bauer et al. (12) 2000 benign and 115 malignant lesions were examined and dermoscopy results were to a great extent comparable to histopathological results. Furthermore, melanocytic nevi were found to be associated with melanoma in that study. Current study included 300 participants with melanocytic nevi, 15 of which were considered malignant and examined histopathologically. Dermoscopic examination was found to provide high specificity in the assessment of these lesions.

On the other hand, some reports have suggested that ABCD scoring may actually be associated with low specificity. In a retrospective study by Pizzichietta et al. (13) a total of 129 melanocytic lesions smaller than $1 \mathrm{~cm}$ in diameter were assessed and $A B C D$ algorithm was reported to be ineffective in the assessment of small melanocytic lesions.

According to the conclusions of international dermoscopy consensus meeting, first organized by the Austrian dermatologist Soyer and followed subsequently by an online convention of 40 leading specialists in 2001, three very important diagnostic criteria for malign melanoma have been proposed as follows: asymmetry, atypical pigment network, and blue-white structures (14). Since this was developed as a screening method for early identification of melanoma, it has a high sensitivity. Presence of at least two of these three criteria is considered to show the presence of malignancy. The 3-point checklist has been rather conceived as a screening test. In a study involving the participation of six inexperienced physicians, a 1-hour training was provided for the 3-point checklist and then these physicians were asked to assess 231 pigmented skin lesions. The rate of melanoma diagnosis rose from $67.9 \%$ with usual clinical examination to $96.3 \%$ with the 3-point checklist (15). In a 2005 study by Soyer et al., (16) 165 melanocytic skin lesions (116 benign, 49 melanoma) were included in the study, and a high rate of diagnosis of malignancy, i.e. $86.7 \%$, was reported, showing the practicability of the 3 -point checklist. Zalaudek et al. (17) also assessed the 3point checklist with the participation of 150 
physician from 29 countries. These authors concluded that experience was required for accurate assessment of the blue-white structures and atypical pigment network. In that study, 3point check list was associated with a sensitivity of 91\% for a diagnosis of melanoma when applied by physician. Similarly, the 3-point checklist was also assessed in our study, and 15 of the 300 melanocytic nevi were considered malignant, with a sensitivity of $97 \%$ based on histopathological correlation. This figure is higher as compared to the reported figures by Zaludek and Argenziano.

Digital dermoscopic devices have also been developed for the assessment of melanocytic lesions, incorporating computer technology, software use, and digital imaging $(18,19)$. These devices have brought important advances in the follow-up and mapping of melanocytic nevi, offering significantly improved practicability for the physicians. In a study from Tuebingen University, 867 melanocytic lesion were assessed using digital dermoscopy of these lesions, and 269 were excised. Histopathology showed the presence benign melanocytic lesions in 185, while 84 had a confirmed diagnosis of melanoma. No excision was considered necessary in 568 lesions digitally assessed as benign, with a diagnostic accuracy of $82 \%$. Thus digital dermoscopic examination was able to provide similar results as those obtained with ABCD criteria, Menzies scoring, as well as the 7-point checklist (20). In the study by Fikrle et al. (21) 260 melanocytic lesions were evaluated using digital dermoscopy, which had a $91 \%$ sensitivity for differentiating between benign and malignant lesions, and $91.3 \%$ sensitivity and $80 \%$ specificity for a diagnosis of malignant melanoma. The authors suggested that digital dermoscopic examination may represent the current diagnostic modality of choice. However, a major limitation of our study is the absence of histopathological correlation in patients who were considered to have malignant lesions based on assessments with this device. In conclusion, a higher rate of diagnosis was found with the use of 3-point checklist in our study as compared to previous reports. In recent years, CASH, 3-point checklist, and 4-point checklist scores have also gained attention as valuable diagnostic tools, as reported by Meo et al., (22) who showed a sensitivity and specificity of $100 \%$ and $76 \%, 60 \%$ and $89 \%$, and $83 \%$ and $82 \%$ for CASH, 3 -point checklist, and $4-$ point checklist scores, respectively. Thus, CASH algorithm was also considered as an effective diagnostic modality.
In a leading 2016 manuscript prepared by a group of leading investigators in the field of dermoscopy, major diagnostic algorithms have been examined. Accordingly, the scoring system with the highest sensitivity was Menzies scoring system, while the 3-point checklist scoring had the lowest sensitivity. Regarding the specificity, $\mathrm{ABCD}$ had the highest specificity as compared to "Chaos and Clues" having the lowest specificity (23). In conclusion, although 3-point checklist scoring and automated screening systems appear to be important diagnostic tools, they also have significant disadvantages. Nevertheless, they also provide certain advantages in terms of the ease of use and duration of assessment. We hope that dermoscopy will soon be able to provide a similar diagnostic yield as histopathology. But certainly further studies are warranted.

Conflict of interest statement: The authors declare that there are no conflicts of interest.

Financial Disclosure: This work was supported by Research Fund of the Van Yuzuncu Yil University. Project Number: 2008-TFU-050.

\section{References}

1. Burgdorf WHC, Plewig G, Wolff HH, Landthaler M. Braun-Falco's Dermatology, 3rd ed., 2009, Springer Verlag; 1403-1413.

2. Aydemir HE. Andrew's Deri Hastalıkları, Klinik Dermatoloji. Melanositik nevüsler ve neoplazmlar. Çeviri kitabı. İstanbul Medikal Yayınc1l1k, 10. Bask1 2008; 685-701.

3. Wolff Klaus, Lowell A. Goldsmith, Stephen I. Katz, Barbara A. Gilchrest, Amy S. Paller, David J. Leffell. Fitzpatrick's Dermatology in General Medicine 7 th ed. The McGraw-Hill Companies. 2008: 122; 1137-1196.

4. Oğuz O. Melanosit hastalıkları, Pediatrik Dermatoloji Kitab1; Nobel Kitapevi: İstanbul 2005: Sayfa 305-317.

5. Onsun N. Kutanöz melanom. Türkiye Klinikleri Dermatoloji Dergisi 2007; 3: 44-50.

6. Tüzün Y, Gürer MA, Serdaroğlu S, Oğuz O, Aksungur LV. Deri tümörleri. Dermatoloji kitabi. Nobel t1p kitapevi 3 bask1 İstanbul 2008; 2: 17591762.

7. Dennis LK, White E, Lee JA, et al. Constitutional factors and sun exposure in relation to nevi: a population-based cross-sectional study. Am J Epidemiol 1996; 143: 248-256.

8. Kuşku E, Lebe B, Soyal MC, et al. Evaluatıon of dermatoscopic and histopathologic features of nevus nevocellularis and of the correlations between these features. Turkiye Klinikleri J Dermatol 2006; 16: 1-6. 
9. Stolz W, Riemann A, Cognetta AB, Pillet L. ABCD rule of dermoscopy: a new practical method for early recognition of malignat melanoma. Eur J Dermatol 1994; 4: 521-527.

10. Akay BN. Algorithms in dermoscopic diagnosis. Turkiye Klinikleri J Int Med Sci 2007; 3: 1-4.

11. Carli P, De Giorgi V, Soyer HP, et al. Dermatoscopy in the diagnosis of pigmented skin lesions: a new semiology for the dermatologist. J Eur Acad Dermatol Venerol 2000; 14: 353-369.

12. Bauer J, Metzler G, Rassner G, Garbe C, Blum A. Dermatoscopy turns histopathologist's attention to the suspicious area in melanocytic lesions. Arch Dermatol 2001; 137: 1338-1340.

13. Pizzichetta MA, Talamini R, Piccolo D, et al. The $\mathrm{ABCD}$ rule of dermatoscopy does not apply to small melanocytic skin lesions. Arch Dermatol 2001; 137: 1376-1378.

14. Argenziano G, Soyer HP, Chimenti S. Dermoscopy of pigmented skin lesions: Results of a consensus meeting via the internet. J Am Acad Dermatol 2003; 48: 679-693.

15. Boyvat A. Three point checklist and seven point checklist in dermoscopic diagnosis. Turkiye Klinikleri J Int Med Sci 2007; 3: 5-9.

16. Soyer HP, Argenziano G, Zalaudek I, et al. Three-point checklist of dermoscopy. A new screening method for early detection of melanoma. Dermatology 2004; 208: 27-33.

17. Zalaudek I, Argenziano G, Soyer HP, et al. Three- point checklist of dermoscopy: An open internet study. Br J Dermatol 2006; 154: 431-437.
18. Gereli MC, Onsun N, Atilganoglu U, Demirkesen C. Comparison of two dermoscopic techniques in the diagnosis of clinically atypical pigmented skin lesions and melanoma: seven-point and threepoint checklists. Int J Dermatol. 2010; 49: 33-38.

19. Özdemir F. Melanom tanıs1. Türkderm 2007; 41 özel say1 2: 6-14.

20. Blum A, Luedtke H, Ellwanger U, et al. Digital image analysis for diagnosis of cutaneous melanoma. Development of a highly effective computer algorithm based on analysis of 837 melanocytic lesions. Br J Dermatol 2004; 151: 1029-1038.

21. Fikrle T, Pizinger K. Digital computer analysis of dermatoscopical images of 260 melanocytic skin lesions; perimeter/area ratio for the differentiation between malign melanomas and melanocytic nevi. J Eur Acad Dermatol Venerol 2007; 21: 48-55.

22. di Meo N, Stinco G, Bonin S, et al. CASH algorithm versus 3-point checklist and its modified version inevaluation of melanocytic pigmented skin lesions: The 4-point checklist. J Dermatol. 2016; 43: 682-685.

23. Carrera C, Marchetti MA, Dusza SW, et al. Validity and Reliability of Dermoscopic Criteria Used to Differentiate Nevi From Melanoma: A Web-Based International Dermoscopy Society Study. JAMA Dermatol 2016; 152: 798-806. 